\title{
OFICINA DE CONOCIMIENTO ABIERTO: UN MODELO PARA INSTITUCIONALIZAR EL ACCESO ABIERTO EN LAS UNIVERSIDADES
}

\author{
Alejandra Nardi y Lucas Yrusta
}
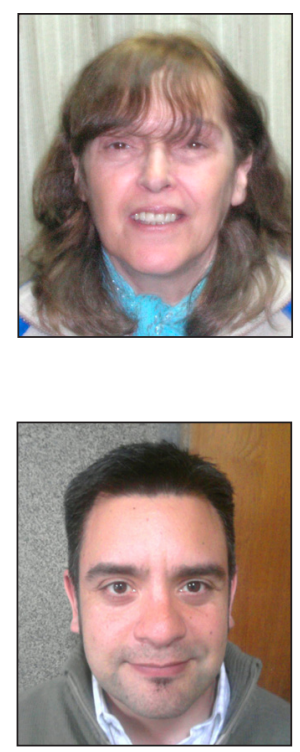

Lucas Yrusta es bibliotecólogo y posgraduado en gestión cultural por la Universidad Nacional de Córdoba (UNC). Es responsable del área de proyectos de la biblioteca de la Fac. de Ciencias Económicas, (UNC). Investigador en los proyectos: Desarrollo e implementación de la colección digital de la UNC (UNC y Univ. Complutense de Madrid). Coordinador operativo de la Oficina Técnica del repositorio institucional de la UNC. Capacitador para el Programa conectar igualdad y la Dirección General de Educación Superior sobre Repositorios Digitales de Objetos de Aprendizaje. http://orcid.org/0000-0001-6386-1172

Alejandra Nardi, licenciada en bibliotecología y documentación, es directora de la Escuela de Bibliotecología y profesora adjunta a cargo de cátedra de la Fac. de Filosofía y Humanidades de la Universidad Nacional de Córdoba (UNC). Es responsable Iberoamericana del Proyecto C/020555/08, Desarrollo e implementación de la colección digital de la UNC (UNC y Univ. Complutense de Madrid). Coordinadora del Programa de formación para desarrollar competencias transversales ( $M$ o de Educación de la Nación, Secretaría de Políticas Universitarias) y coordinadora general del repositorio institucional de la UNC.

http://orcid.org/0000-0002-6451-4858

anardi@eco.unc.edu.ar

lucasyrusta@gmail.com

Univ. Nacional de Córdoba, Fac. de Ciencias Económicas Avda. Valparaíso, s/n. Ciudad Universitaria

5000 Córdoba, Argentina

\section{Resumen}

Se plantea un modelo de gestión para la creación de una Oficina de Conocimiento Abierto (OCA) en la Universidad Nacional de Córdoba (UNC), Argentina, definida para asesorar y capacitar a los miembros que integran los diferentes proyectos sobre acceso abierto iniciados dentro del ámbito universitario. Su función es contribuir a mejorar el sistema de comunicación científica y dar visibilidad a la producción intelectual generada por la comunidad universitaria y a los fondos antiguos localizados en sus bibliotecas, museos y archivos. Se detallan los antecedentes y se describen la misión, visión, objetivos, estructura y funciones principales de la Oficina.

\section{Palabras clave}

Acceso abierto, Oficina de Conocimiento Abierto, Modelo de gestión, Universidad Nacional de Córdoba, Argentina

Title: Open Knowledge Office: a model for institutionalizing the open access movement in universities

\section{Abstract}

This paper offers a management model to create an open access office at the Universidad Nacional de Córdoba. This office intends to provide instruction and training to the members of the different open access projects being developed at the University. It aims at improving the scientific communication system and giving visibility to the academic production, as well as the historic resources that belong to the libraries, museums and archives of the University. A detailed description of the mission, vision, objectives, structure and main functions of the open access office are described

\section{Keywords}

Open access, Open knowledge office, Management model, Universidad Nacional de Córdoba, Argentina.

Nardi, Alejandra; Yrusta, Lucas. "Oficina de Conocimiento Abierto: un modelo para institucionalizar el acceso abierto en las universidades". El profesional de la información, 2012, noviembre-diciembre, v. 21, n. 6, pp. 633-637. 


\section{Introducción}

A partir de las decisiones acordadas por el máximo órgano de gobierno de la Universidad Nacional de Córdoba (2010), los responsables de los diferentes proyectos de acceso abierto de la misma comenzaron a llevar a cabo acciones conjuntas, comprometidos con lo institucional y trascendiendo los objetivos propios de las unidades académicas y de las dependencias del Rectorado. Se debatió la estrategia para potenciar el acceso abierto en la comunidad universitaria y se acordó crear una Oficina de Conocimiento Abierto que consolide y haga sustentables todas las iniciativas de acceso abierto en la UNC.

Se efectuó una investigación bibliográfica, para conocer otros modelos de gestión. Se detectó la existencia de la Oficina de Conocimiento Abierto de la Universidad de Salamanca, encontrando coincidencias respecto de la necesidad de constituir en el ámbito universitario una estructura que ordenara los aspectos del acceso abierto. Se coincide con lo expresado en el siguiente párrafo "Una política de impulso al acceso abierto tiene que contemplar un conjunto amplio de mecanismos de intervención como pueden ser la creación de infraestructuras y prestación de servicios, la comunicación y la difusión, la incentivación económica, la coordinación institucional, así como la reglamentación" (Abadal, 2012).

\section{Antecedentes de la Universidad Nacional de Córdoba}

La UNC está localizada en la ciudad de Córdoba, Argentina. Fundada en 1613, es la universidad más antigua del país y la segunda de América Latina. Es de carácter público, en ella se forman 112.186 estudiantes de diversas procedencias, en 271 carreras de grado y posgrado, y cuenta con 11.053 docentes e investigadores. Está conformada por 13 facultades, 98 centros de investigación y servicios, 25 bibliotecas y 16 museos. Anualmente se llevan a cabo 1.500 proyectos de investigación y vinculación (datos actualizados a febrero 2012). En 2013 la UNC cumplirá 400 años, y "asume como marco identitario común la defensa de la educación pública, la democratización de los procesos educativos y la ampliación de las fronteras universitarias hacia los sectores que aún están excluidos de esta comunidad" (UNC, 2012).

\section{Antecedentes de acceso abierto en la UNC}

En septiembre de 2008, las bibliotecas de la UNC junto con la biblioteca de la Universidad Complutense de Madrid, se presentan a la convocatoria de la Agencia Española de Cooperación Internacional para el Desarrollo (Aecid), Programa de Cooperación Internuniversitaria (PCI), con el proyecto: Desarrollo e implementación de la colección digital de la UNC. Esta acción complementaria sería el precedente de un segundo proyecto: Fortalecimiento institucional de la dimensión internacional de la UNC. Su objetivo es fortalecer a la UNC en su dimensión estratégica de internacionalización universitaria y cooperación al desarrollo. En este marco, uno de los objetivos específicos se definió con el propósito de preservar y dar visibilidad internacional a parte de las colecciones históricas y a la producción intelectual generada por los investigadores de la Universidad.
Las acciones desarrolladas y concretadas a septiembre de 2012, son:

- creación del Repositorio Digital Universitario (RDU), destinado a albergar la producción científica, académica y cultural; para su implementación se utiliza DSpace. http://rdu.unc.edu.ar

- Portal de revistas de la UNC, que se implementa con el programa Open Journal Systems.

http://revistas.unc.edu.ar

La digitalización de la Librería Jesuítica de la Biblioteca Mayor y la colección Monseñor Pablo Cabrera de la Facultad de Filosofía y Humanidades (FFYH), son el primer paso para conformar la Biblioteca Histórica Digital de la UNC.

En noviembre de 2008 se crea el sitio OCW (OpenCourseWare)-UNC, dependiente de la Secretaría de Asuntos Académicos. Su objetivo es compartir los recursos educativos en abierto.

En diciembre de 2010, el Honorable Consejo Superior de la UNC, crea la Comisión Asesora a cargo del Programa Integral de Tecnologías de Información y Comunicación, con el objetivo de "analizar y desarrollar estrategias tendentes a fortalecer y promover el acceso libre y sin restricciones al conocimiento" (UNC, 2010). La UNC asume un compromiso con el movimiento internacional de acceso abierto, entendiendo que la producción generada en una universidad pública debe estar disponible y accesible para toda la sociedad, por ser un recurso público y social.

El 29 de noviembre de 2011 se inaugura el repositorio de materiales educativos para la formación y el desempeño docente Ansenuza, elaborado por el Área de Tecnología Educativa de la FFYH y la Dirección General de Educación Superior (DGES). El repositorio se enmarca en el proyecto Universidad en la sociedad del conocimiento. Fortalecimiento institucional de áreas dedicadas a la enseñanza universitaria con tecnologías, en cooperación con la Universidad de Santiago de Compostela, y financiado por la Aecid. El modelo de gestión de Ansenuza es similar al del $R D U$, ya que cuenta con nodos (escuelas e institutos de educación) representativos en las instituciones de la DGES. Ambos repositorios trabajan con la misma plataforma DSpace, comparten los mismos problemas, mejoras e implementaciones.

En 2012 han comenzado otras dos iniciativas:

a) creación del Repositorio del Observatorio Astronómico de Córdoba;

b) proyecto de apoyo a la informatización de los archivos documentales y colecciones del Museo de Antropología $(F F Y H)$.

\section{Creación de la Oficina de Conocimiento Abierto}

A continuación se describen, la misión, visión, objetivos, organigrama y funciones de la oficina.

\section{Misión}

Brinda asesoramiento y capacitación a los miembros que integran los diferentes proyectos sobre acceso abierto iniciados dentro del ámbito universitario. Las acciones que 


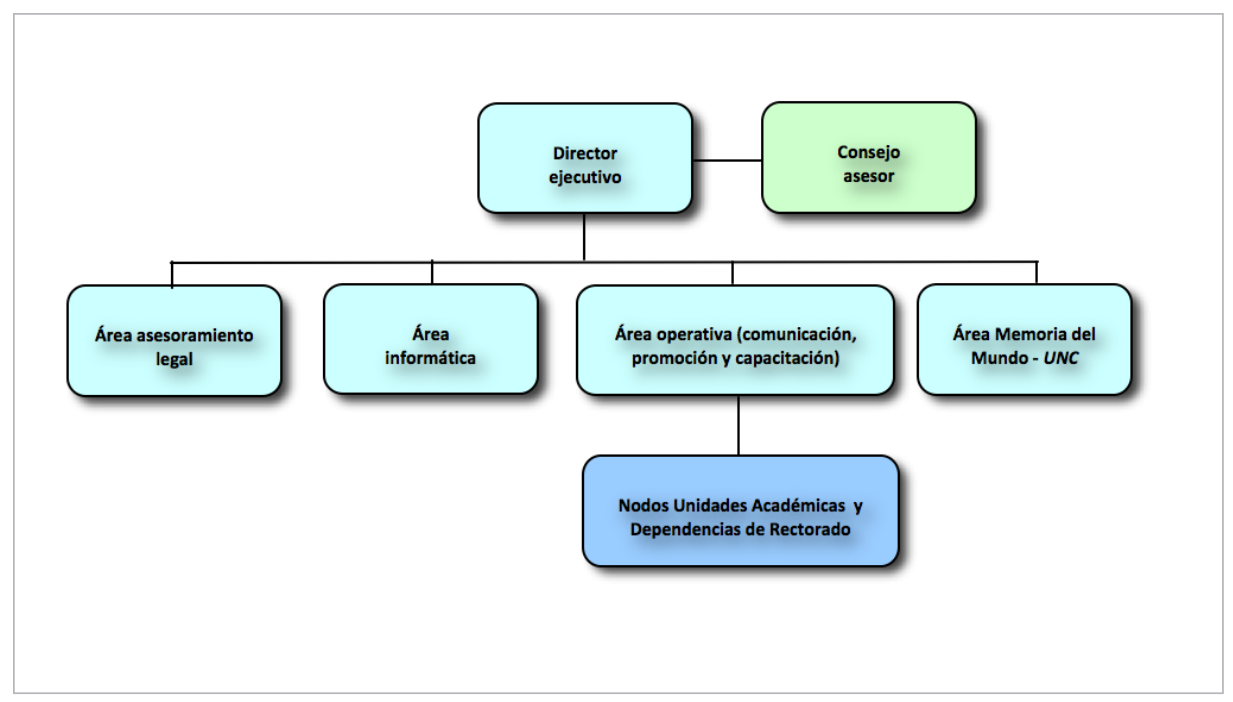

Organigrama de la Oficina de Conocimiento Abierto - UNC universidad más antigua de América Latina, ajustándose a las pautas de la Unesco en el documento: Memoria del mundo. Directrices para la salvaguardia del patrimonio documental (Edmondson, 2002).

- Proporcionar la infraestructura tecnológica para dar soporte a un espacio que permita la preservación y difusión de la producción digital y de las colecciones históricas, basada en software libre, estándares abiertos y normas de accesibilidad. emprende tienen el propósito de contribuir a mejorar el sistema de comunicación científica y dar visibilidad a la producción intelectual generada por la comunidad universitaria y a los fondos antiguos localizados en sus bibliotecas, museos y archivos. Utiliza para su implementación estándares de interoperabilidad y programas informáticos de código abierto. Los beneficiarios de sus acciones son la propia Universidad, la comunidad académica internacional y la sociedad en general.

\section{Visión}

La UNC pretende formar parte activa del movimiento internacional de archivos abiertos, integrándose al resto de los países del mundo con el lema: "La ciencia y sus resultados son un recurso público y social". Aspira a ser un referente para América Latina, contribuyendo a la democratización de la información y del conocimiento.

\section{Objetivos}

- Definir las políticas institucionales sobre el acceso, difusión y preservación de la información científica y cultural generada por la comunidad universitaria y de los fondos antiguos albergados en la UNC.

- Crear una cultura de acceso abierto en la UNC, y concienciar a la comunidad universitaria de que "la investigación financiada con fondos públicos debe gozar de amplia difusión a través de la publicación en acceso abierto de datos y documentos científicos" (UE, 2012).

- Incrementar la visibilidad de la producción educativa, científica y cultural de la UNC.

- Promover la creación y difusión de conocimientos producidos colaborativamente entre distintos actores socioeducativos.

- Apoyar y promover la edición de revistas científicas en formato digital y de acceso abierto, así como contribuir a la mejora de la calidad académica de las mismas.

- Impulsar la creación de una Biblioteca Histórica Digital para preservar los fondos antiguos y asegurar su disponibilidad permanente ofreciendo a los investigadores acceso directo e inmediato.

- Recuperar y mantener viva la memoria de la segunda

\section{Organigrama y funciones de la Oficina de Conoci- miento Abierto}

\section{Director ejecutivo}

- Generar políticas en forma conjunta con el Consejo Asesor que favorezcan la sostenibilidad de los repositorios digitales de la UNC.

- Definir políticas en forma conjunta con el Consejo Asesor dirigidas a garantizar el respeto por los derechos de los autores de la producción científica, tecnológica y cultural de la UNC.

- Promover el acceso abierto a la producción científica, académica y cultural de la UNC.

- Potenciar el movimiento de acceso abierto en la UNC.

- Representar a la UNC en el Sistema Nacional de Repositorios Digitales (SNRD) dependiente del Ministerio de Ciencia, Tecnología e Innovación Productiva.

- Definir un conjunto de indicadores para medir el impacto de artículos individuales, atendiendo las nuevas recomendaciones de la Iniciativa Acceso Abierto de Budapest.

\section{Área de asesoramiento legal}

- "Orientar a los investigadores sobre cómo dar cumplimiento a las políticas de acceso abierto, especialmente a la hora de gestionar los propios derechos de propiedad intelectual, con el fin de facilitar acceso abierto a sus publicaciones" (UE, 2012).

\section{Área de informática}

- Establecer mecanismos para garantizar la preservación de la colección digital, siendo su fin último, "asegurar que la información permanezca inalterable, utilizable, legible y accesible en las diferentes plataformas tecnológicas que vayan surgiendo" (CRID, 2012).

- Mantener la infraestructura informática que soporta los repositorios de la UNC y asegurar su accesibilidad y actualización.

- Asesorar a los nodos (bibliotecas) de las Unidades Académicas y dependencias del Rectorado, para establecer e implementar los medios técnicos apropiados para la creación de las versiones electrónicas de la información científica y cultural generada por la comunidad universitaria. 
- Trabajar en forma colaborativa con la Oficina de Inclusión Educativa de Personas en Situación de Discapacidad, en la selección de las tecnologías más apropiadas.

- Representar a la UNC en el Sistema Nacional de Repositorios Digitales (SNRD), en calidad de responsable informático.

\section{Área operativa}

\section{Coordinador operativo}

- Planificar las actividades entre las áreas que componen la Oficina de Conocimiento Abierto.

- Coordinar y programar actividades en forma conjunta con los nodos pertenecientes a las diferentes Unidades Académicas y dependencias del Rectorado.

- Definir, evaluar y optimizar los servicios ofrecidos por la Oficina.

- Planificar el Programa de Capacitación Continua sobre acceso abierto destinado a los diferentes estamentos de la Universidad.

- Proponer y planificar la integración con redes de repositorios a nivel nacional, regional e internacional.

- Coordinar acciones de comunicación y difusión de la Oficina.

- Asesorar a los editores de las revistas académicas, científicas y culturales de la Universidad.

- Representar a la UNC en el Sistema Nacional de Repositorios Digitales (SNRD), en calidad de responsable técnico.

\section{Responsable de comunicación}

- Comunicar novedades y eventos sobre las actividades propuestas por la Oficina a la comunidad universitaria y a la sociedad en general.

- Participar en la organización de reuniones, programando la agenda de la Oficina.

- Definir los canales y espacios de comunicación para la interacción entre los integrantes de la Oficina, los nodos y los editores.

- Realizar estudios de opinión con la finalidad de conocer la autopercepción de la comunidad universitaria acerca del movimiento de archivos abiertos.

Responsable de capacitación

- Diseñar y gestionar los talleres y cursos relacionados con diversos aspectos del acceso abierto.

- Planificar conjuntamente con al área de comunicación, tutoriales y materiales didácticos que fortalezcan el proyecto de acceso abierto.

La investigación financiada con fondos públicos debe gozar de amplia difusión a través de la publicación en acceso abierto de datos y documentos científicos (UE, 2012)

\section{Responsable de estandarización}

- Coordinar un grupo de especialistas para analizar los distintos esquemas de metadatos existentes, su interoperabilidad y su aplicación.

- Redactar un manual de buenas prácticas que sirva de guía y orientación para el cumplimiento de los estándares propuestos.

- Trabajar e integrar equipos interdisciplinares con otras instituciones, para aumentar la potencialidad e interoperabilidad de los repositorios locales y regionales.

\section{Área Memoria del Mundo UNC}

- Crear una mayor conciencia de la existencia e importancia del patrimonio documental.

- Acordar con los directores de bibliotecas, museos y archivos acciones conjuntas para mantener viva la memoria de la UNC.

- Planificar e implementar acciones y estrategias para preservar en soporte digital el patrimonio cultural de la UNC localizado en bibliotecas, archivos y museos.

- Facilitar el acceso universal al patrimonio documental de la UNC.

- Integrar y participar activamente del Programa $\mathrm{Me}$ moria del Mundo de la Organización de las Naciones Unidas para la Educación la Ciencia y la Cultura (Unesco), y en otros programas y redes vinculados a la preservación de los patrimonios documentales. 


\section{Conclusiones}

La institucionalización de una Oficina de Conocimiento Abierto en la Universidad Nacional de Córdoba permitirá consolidar e integrar las diferentes iniciativas que han surgido en forma espontánea en las unidades académicas y dependencias del rectorado.

La institucionalización de la Oficina potenciará una cultura de acceso abierto en la UNC, y concienciará a la comunidad universitaria acerca de la importancia de que "la investigación financiada con fondos públicos debe gozar de amplia difusión a través de la publicación en acceso abierto de datos y documentos científicos" (UE, 2012)

\section{Bibliografía}

Abadal, Ernest. Acceso abierto a la ciencia. Colección El profesional de la información, n. 5. Barcelona: Editorial UOC, 2012. ISBN 9788497885485

http://diposit.ub.edu/dspace/bitstream/2445/24542/1/262142. $p d f$

Budapest Open Access Initiative. Diez años desde la Budapest Open Access Initiative: hacia lo abierto por defecto. http://www.soros.org/openaccess/boai-10-translations/ spanish

Centro Regional de Información sobre Desastres para América Latina y el Caribe, CRID. Toolkit para la creación de productos y servicios de información sobre riesgo y desastres, 2012.

http://toolkit.cridlac.org/modulo-1-planificacion-ygerencia-de-un-centro-de-informacion-en-gestion-delriesgo-de-desastres

Edmondson, Ray. Memoria del mundo, directrices para la salvaguardia del patrimonio documental. Ed. rev. París: Unesco, 2002. $p d f$

Organización de las Naciones Unidas para la Educación la Ciencia y la Cultura. Programa Memoria del Mundo.

http://www. unesco.org/new/es/communication-andinformation/flagship-project-activities/memory-of-theworld

Unión Europea. "Recomendación de la Comisión de 17 de julio de 2012 relativa al acceso a la información científica y a su preservación, 2012/417/UE". Diario Oficial de la Unión Europea, 2012, julio, pp. 39-43.

http://eur-lex.europa.eu/LexUriServ/LexUriServ.do?uri=OJ:L :2012:194:0039:0043:ES:PDF

Universidad Nacional de Córdoba. Secretaría de Asuntos Académicos. Oficina de Graduados. Hacia los 400 años de la universidad nacional de Córdoba.

http://www.unc.edu.ar/seccion/novedades/2012/abril/ unc-saa-graduarte-anexo-informativo.pdf

Universidad de Salamanca. ¿Qué es la Oficina de Conocimiento Abierto?

http://oca.usal.es/index.php?option=com_content\&task=vi ew\&id=30\&/temid $=105$ 


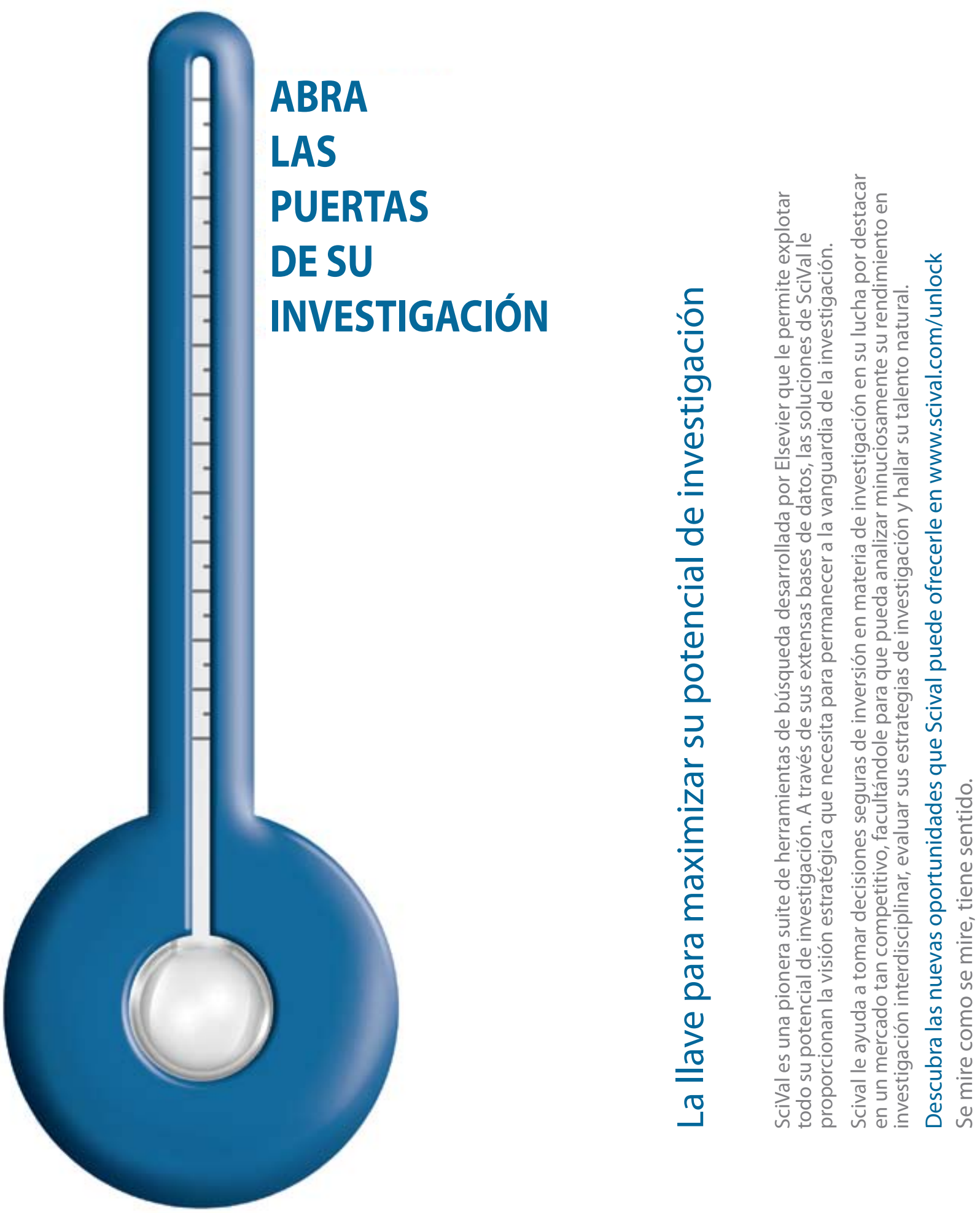

Scival

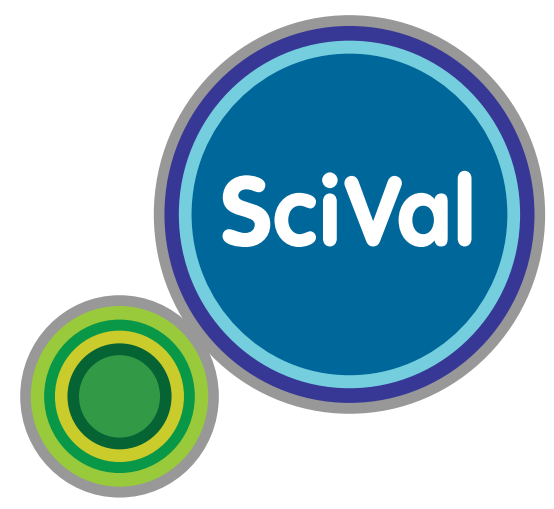

